\title{
The Extension of Cauchy Integral Formula to the Boundaries of Fundamental Domains
}

\author{
Dorin Ghisa \\ Glendon College, York University, Toronto, Canada \\ Email:dghisa@yorku.ca
}

How to cite this paper: Ghisa, D. (2020) The Extension of Cauchy Integral Formula to the Boundaries of Fundamental Domains. Advances in Pure Mathematics, 10, 181-199.

https://doi.org/10.4236/apm.2020.104012

Received: March 1, 2020

Accepted: April 24, 2020

Published: April 27, 2020

Copyright (อ 2020 by author(s) and Scientific Research Publishing Inc. This work is licensed under the Creative Commons Attribution International License (CC BY 4.0).

http://creativecommons.org/licenses/by/4.0/

\section{(c) (i) Open Access}

\begin{abstract}
The Cauchy integral formula expresses the value of a function $f(z)$, which is analytic in a simply connected domain $D$, at any point $z_{0}$ interior to a simple closed contour $C$ situated in $D$ in terms of the values of $\frac{f(z)}{z-z_{0}}$ on $C$. We deal in this paper with the question whether $C$ can be the boundary $\partial \Omega$ of a fundamental domain $\Omega$ of $f(z)$. At the first look the answer appears to be negative since $\partial \Omega$ contains singular points of the function and it can be unbounded. However, the extension of Cauchy integral formula to some of these unbounded curves, respectively arcs ending in singular points of $f(z)$ is possible due to the fact that they can be obtained at the limit as $r \rightarrow \infty$ of some bounded curves contained in the pre-image of the circle $|z|=r$ and of some circles $|z-a|=1 / r$ for which the formula is valid.
\end{abstract}

\section{Keywords}

Fundamental Domain, Dirichlet Functions, Modular Function, Weierstrass $\wp$ Function

\section{Introduction}

We make reference to [1] for elementary knowledge in complex analysis used below. It is known (see [2]) that for every rational function $R(z)$ of degree $n$ the complex plane can be partitioned into $n$ sets whose interior are fundamental domains of $R(z)$, i.e. they are mapped conformally (hence bijectively) by $R(z)$ onto the whole complex plane with some slits. A similar partition takes place for transcendental functions (see [3]), except that for those functions the 
number of fundamental domains is infinite. Every fundamental domain $\Omega$ of an analytic function $f(z)$ is either unbounded or $\Omega \cup \partial \Omega$ contains singular points of $f(z)$, or both.

Although integrals on unbounded contours have been used frequently in complex analysis (see [1], page 214), they have never appeared in the context of Cauchy integral formula. The main novelty of this paper is that it makes possible such an undertaking. The famous Cauchy integral formula is in this way upgraded from a rather local instrument to a more global one. Moreover, it shows that the functions we are studying are completely determined by the values on the boundaries of their fundamental domains.

The integral on $\partial \Omega$ of $\frac{f(z)}{z-z_{0}}$ shall be treated as an improper integral the convergence of what remains to be investigated. This can be accomplished in different ways which apply to particular classes of functions; hence instead of trying to prove theorems valid for any analytic function, we must treat separately those classes of functions. However, the techniques used are in general similar; namely they consist in isolating the singular points and $z=\infty$ by the pre-image of some circles whose radii are let tend to zero, respectively to infinity, then in applying the Cauchy integral formula to the bounded sub-domains of $\Omega$ obtained in this way and making sure that the integrals on the boundaries of the complementary domains tend to zero when the radii tend to zero or to infinity. As $f(z)$ is injective in every fundamental domain, if such a domain is mapped conformally by the function onto the complex plane with a slit; then for some values $r>0$ there is a function $\varphi\left(r \mathrm{e}^{\mathrm{i} \theta}\right)$ corresponding to that fundamental domain such that $f\left(\varphi\left(r \mathrm{e}^{\mathrm{i} \theta}\right)\right)=r \mathrm{e}^{\mathrm{i} \theta}, 0 \leq \theta \leq 2 \pi$. The function $\varphi\left(r \mathrm{e}^{i \theta}\right)$ is injective in the interval $[0,2 \pi)$ and maps this interval onto an arc $\gamma$ included in that fundamental domain. Making the change of variable $z=\varphi\left(r \mathrm{e}^{\mathrm{i} \theta}\right)$ in the integral $\int_{\gamma} \frac{f(z)}{Z-z_{0}} \mathrm{~d} z$ it becomes an integral on the interval $[0,2 \pi)$ and it is possible that it tends to zero as $r \rightarrow \infty$ or $r \rightarrow 0$. This assertion should be checked for every particular class of functions.

The contours we used for integration needed to be illustrated and most of the graphics are computer generated by the software Mathematica. When this was not possible, we used illustration by hand made drawings. However, they are pictures of known fundamental domains (see [1], page 268 and 282). One of the most studied classes of meromorphic functions is that of Dirichlet functions and it can be considered as a prototype in many aspects. Let us start then with this class.

\section{General Properties of Dirichlet Functions}

The Dirichlet functions are obtained by analytic continuation of general Dirichlet series across the line of convergence. The family of general Dirichlet series includes that of well known Dirichlet L-series defined by Dirichlet characters. These last series can be all extended as meromorphic functions in the whole 
complex plane. The extended functions are called Dirichlet L-functions. They are implemented in Mathematica and some affirmations about general Dirichlet functions are illustrated by using Dirichlet L-functions. However, the interest in more general functions is obvious and we have recently devoted to them a lot of publications (see [2]-[15]). An account of recent advances in this field can be found in [8].

A general Dirichlet series $\zeta_{A, \Lambda}(s)$ is defined by an arbitrary sequence of complex numbers $A=\left(a_{1}, a_{2}, \cdots\right)$, the coefficients of the series and by a non decreasing sequence of non negative numbers $\Lambda=\left(\lambda_{1}, \lambda_{2}, \cdots\right)$, the exponents of the series. It is given by the formula

$$
\zeta_{A, \Lambda}(s)=\sum_{n=1}^{\infty} a_{n} \mathrm{e}^{-\lambda_{n} s}
$$

We will deal only with normalized general Dirichlet series in which $a_{1}=1$ and $\lambda_{1}=0$. For such a series we have $\lim _{\sigma \rightarrow+\infty} \zeta_{A, \Lambda}(\sigma+i t)=1$ uniformly with respect to $t$ (see [8], Theorem 3). There is a number $\sigma_{c}$, called the abscissa of convergence of $\zeta_{A, \Lambda}(s),-\infty \leq \sigma_{c} \leq+\infty$ such that the series (1) converges for $\operatorname{Re} s>\sigma_{c}$ and it diverges for $\operatorname{Re} s<\sigma_{c}$. The series converges uniformly on compact subsets of $\operatorname{Re} s>\sigma_{c}$ and therefore it is an analytic function in that half plane. Denoting by $\zeta_{A, \mathrm{e}^{\Lambda}}(s)=\sum_{n=1}^{\infty} a_{n} \mathrm{e}^{-\mathrm{e}^{\lambda_{n}}}$ we have proved in [8] that if the abscissa of convergence of $\zeta_{A, \Lambda}(s)$ is finite then the abscissa of convergence of $\zeta_{A, e^{\Lambda}}(s)$ is zero and if $\zeta_{A, e^{\Lambda}}(s)$ has only isolated singular points on $\operatorname{Re} s=0$, then $\zeta_{A, \Lambda}(s)$ can be continued across the line $\operatorname{Re} s=\sigma_{c}$ to a meromorphic function in the whole complex plane. We keep the notation $\zeta_{A, \Lambda}(s)$ for the extended function when it exists and we call it Dirichlet function. Following Speiser [16], who studied the Riemann Zeta function, we have used in [2]-[15] the pre-image of the real axis by $\zeta_{A, \Lambda}(s)$. This is the set of points in the $s$-plane where $\zeta_{A, \Lambda}(s)$ takes real values. For every Dirichlet function it is a family of analytic curves whose structure has very profound implications on the value distribution of that function. Figure 1(a) illustrates the pre-image of the real axis by a Dirichlet L-function defined by a complex Dirichlet character and Figure 1(b) by a real one. Details about Figure 1(c) are found in Section 3.

We have proved (see for example [8]) that for any Dirichlet function $\zeta_{A, \Lambda}(s)$ this pre-image is formed with unbounded curves (components) which fall into three categories. Namely, there are infinitely many curves $\Gamma_{k}^{\prime}, k \in \mathbb{Z}$, which do not intersect each other and consecutive $\Gamma_{k}^{\prime}$ and $\Gamma_{k+1}^{\prime}\left(\Gamma_{k}^{\prime}\right.$ below $\left.\Gamma_{k+1}^{\prime}\right)$ form infinite strips $S_{k}$ extending for $\operatorname{Re} s$ going from $-\infty$ to $+\infty$. The counting is such that $0 \in S_{0}$. Every curve $\Gamma_{k}^{\prime}$ is mapped homeomorphycally by $\zeta_{A, \Lambda}(s)$ onto the interval $(1,+\infty)$ of the real axis and therefore every $S_{k}$ -strip is mapped (not necessarily one to one) onto the whole complex plane with a slit alongside this interval. For $k \neq 0$ every strip $S_{k}$ contains a unique component $\Gamma_{k, 0}$ of the pre-image of the real axis which is mapped homeomorphycally by $\zeta_{A, \Lambda}(s)$ onto the interval $(-\infty, 1)$ of the real axis and a finite number of components $\Gamma_{k, j}, j \neq 0$ which are mapped each one homeomorphycally by $\zeta_{A, \Lambda}(s)$ onto the whole real axis. The component $\Gamma_{k, 0}$ 


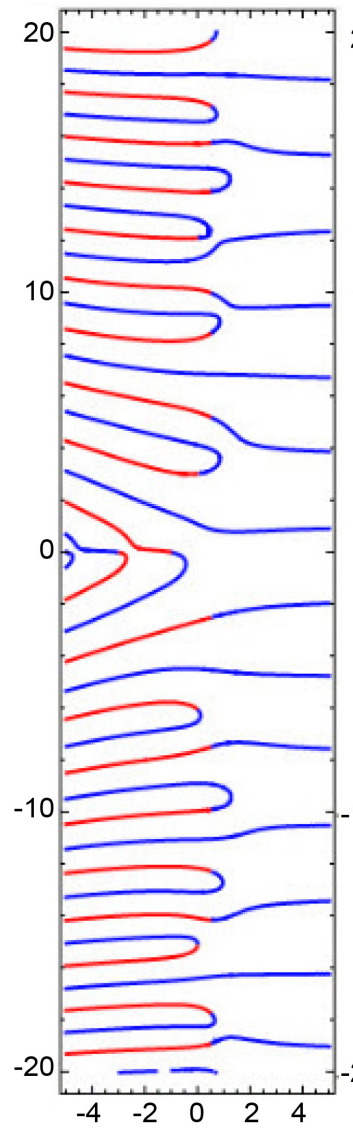

(a)

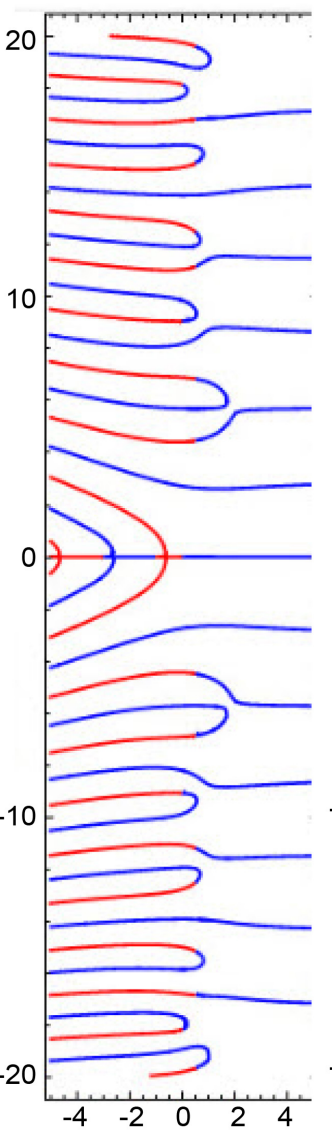

(b)

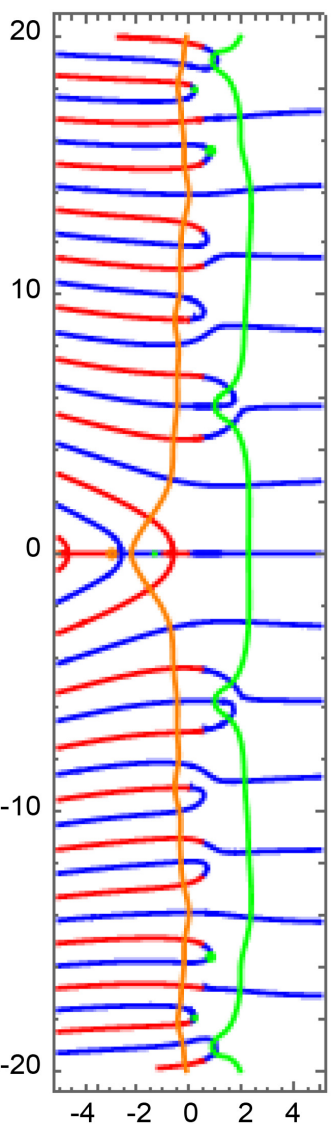

(c)

Figure 1. The pre-image of the real axis by Dirichlet L-functions.

extends for Res going from $-\infty$ to $+\infty$, while $\Gamma_{k, j}, j \neq 0$ are parabola shaped curves with a finite supremum of $\operatorname{Re} s$, therefore we can distinguish the interior and the exterior of such a curve.

In the case of a strip $S_{k}, k \neq 0$, if $\zeta_{A, \Lambda}^{\prime}\left(v_{k, j}\right)=0$ then connecting $\zeta_{A, \Lambda}\left(v_{k, j}\right)$ with $z=1$ by a Jordan arc $\eta_{k, j}$ the component of the pre-image of $\eta_{k, j}$ passing through $v_{k, j}$ can be an unbounded curve $\gamma_{k, j}$, when for $\sigma+i t \in \gamma_{k, j}$ we have $\lim _{\sigma \rightarrow+\infty} \zeta_{A, \Lambda}(\sigma+i t)=1$. On the other hand the origin of such a curve must be a point $u_{k, j}$ on a curve $\Gamma_{k, j}$ such that $\zeta_{A, \Lambda}\left(u_{k, j}\right)=1$. The curve $\gamma_{k, j}$ is bounded when its ends belong to different curves $\Gamma_{k, j}$ and $\Gamma_{k, j^{\prime}}$. This is the case when $\Gamma_{k, j}$ and $\Gamma_{k, j^{\prime}}$ are embraced curves (see [8]) and when $k=0$. The curve $\gamma_{k, j}$ is mapped 2 to 1 by $\zeta_{A, \Lambda}(s)$ onto $\eta_{k, j}$. Then we can form fundamental domains using parts of the curves $\Gamma_{k, j}$, the curves $\gamma_{k, j}$ (and $\Gamma_{k}^{\prime}$, when is the case, as in Figure 2). These are strips unbounded to the right and to the left when $\gamma_{k, j}$ is unbounded and they are bounded to the right when $\gamma_{k, j}$ is bounded. They are mapped conformally by $\zeta_{A, \Lambda}(s)$ onto the whole complex plane with some slits alongside the interval $[1,+\infty)$ of real axis and some other slits alongside $\eta_{k, j}$.

In the case of the strip $S_{0}$, when the zeros of $\zeta_{A, \Lambda}^{\prime}(s)$ are complex, the curves $\gamma_{0, j}$ are all bounded for $j \neq 0$ and together with $\Gamma_{k}$ they form the 

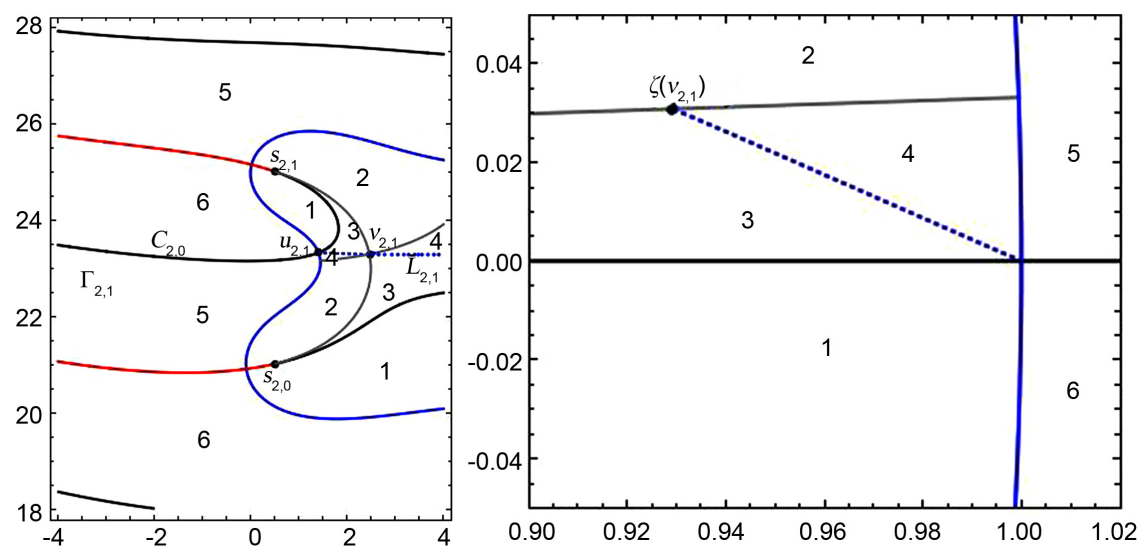

Figure 2. Conformal mapping of fundamental domains by $\zeta(s)$.

boundaries of fundamental domains bounded to the right. It is known (see, for example [13]) that every $S_{k}$-strip, $k \neq 0$ of $\zeta_{A, \Lambda}(s)$ can be partitioned into a finite number of sets whose interior are fundamental domains of $\zeta_{A, \Lambda}(s)$. The $S_{0}$-strip contains infinitely many fundamental domains. The way they are mapped conformally onto the complex plane with some slits by the Riemann Zeta function is illustrated in Figure 2 (see [13], Figure 6).

\section{Cauchy Integral Formula for Fundamental Domains and $S_{k}$-Strips of the Function $\zeta_{A, \Lambda}(s)$}

The Cauchy integral formula has the form:

$$
f\left(z_{0}\right)=\frac{1}{2 \pi i} \int_{C} \frac{f(z)}{z-z_{0}} \mathrm{~d} z
$$

where the function $f(z)$ is analytic in a simply connected domain $D$ containing the simple closed contour $C$ and $z_{0}$ is an arbitrary point inside $C$.

We would like $f(s)$ to be a Dirichlet function $\zeta_{A, \Lambda}(s)$ and $C$ to be the boundary $\partial \Omega$ of a fundamental domain $\Omega$ of $\zeta_{A, \Lambda}(s)$ or the boundary $\partial S_{k}$ of an $S_{k}$-strip. The problem is that $\partial \Omega$ and $\partial S_{k}$ are not simple closed contours. However, we can show that the formula (2) can be extended to these curves.

The shape of the fundamental domains of $\zeta_{A, \Lambda}(s)$ depends on the pre-image of the real axis and on the zeros of $\zeta_{A, \Lambda}^{\prime}(s)$. Since $\zeta_{A, \Lambda}(s)$ is injective in every fundamental domain the zeros of $\zeta_{A, \Lambda}^{\prime}(s)$ must be located on the boundaries of these domains. Figure 3 portrays a fundamental domain $\Omega$ of $\zeta_{A, \Lambda}(s)$ bounded by a curve $\Gamma_{k+1}^{\prime}$, the part of the last curve $\Gamma_{k, j}$ from $S_{k}$ on which $\operatorname{Re} s$ vary from $-\infty$ to $\operatorname{Re} u_{0}$, where we have $\zeta_{A, \Lambda}\left(u_{0}\right)=1$, as well as the pre-image of the segment determined by $z=1$ and $z=\zeta_{A, \Lambda}\left(s_{1}\right)$ where $s_{1}$ is the zero of $\zeta_{A, \Lambda}^{\prime}(s)$ the closest to $u_{0}$. The pre-image $\eta_{1}$ of the circle $\gamma_{1}:|z|=r$ and the pre-image $\eta_{3}+\eta_{6}$ of the circle $\gamma_{3}+\gamma_{6}:|z-1|=\epsilon$ are also drawn, where $r$ is big enough and $\epsilon$ is small enough. Figure 1(c) illustrates computer generated pre-images of these circles for $r=10$ (the orange curve) 

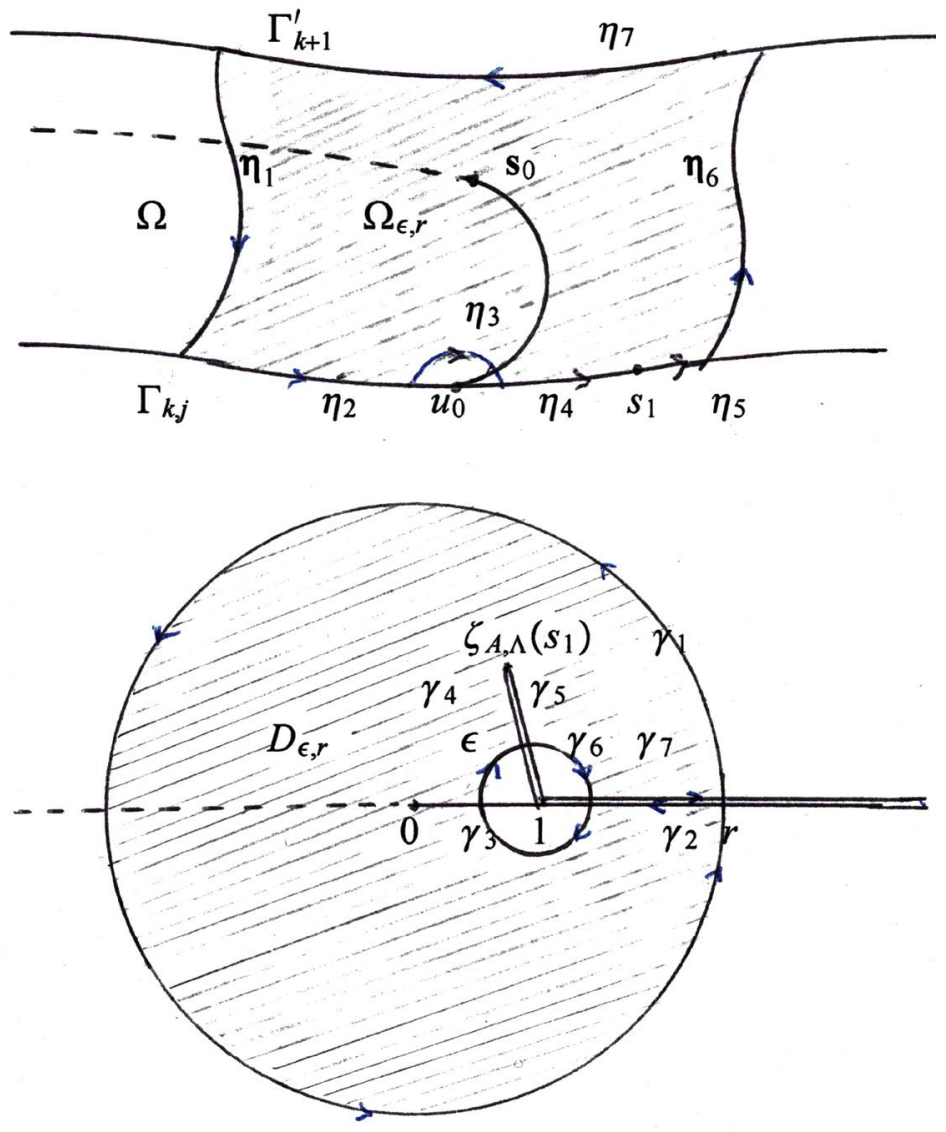

Figure 3. A fundamental domain of $\zeta_{A, \Lambda}(s)$ and its conformal mapping.

and $\epsilon=1 / 10$ (the green curve). It has been worked by Florin Alan Muscutar. Due to the continuity of $\zeta_{A, \Lambda}(s)$ at $s=u_{0}$ and to the fact that $\zeta_{A, \Lambda}(s)$ is a normalized Dirichlet series, the $\operatorname{arc} \eta_{3}$ squeezes to the point $u_{0}$ and $\operatorname{Re} s$ with $s$ on $\eta_{6}$ tends to $+\infty$ as $\epsilon \rightarrow 0$. Also Res with $s$ on $\eta_{1}$ tends to $-\infty$ as $r \rightarrow+\infty$.

The domain $\Omega$ is mapped conformally by $\zeta_{A, \Lambda}(s)$ onto the complex plane with a slit alongside the subinterval $[1,+\infty)$ of the real axis and alongside the segment determined by $z=1$ and $z=\zeta_{A, \Lambda}\left(s_{1}\right)$. Also, the domain $\Omega_{\epsilon, r}$ is mapped conformally onto the ring domain $D_{\epsilon, r}$ determined by the two circles with the corresponding slit (see Figure 3). The function $\zeta_{A, \Lambda}(s)$ is analytic in a domain containing $\Omega_{\epsilon, r} \cup \partial \Omega_{\epsilon, r}$ and therefore the Cauchy integral formula is valid for $\partial \Omega_{\epsilon, r}$.

Theorem 1. If we denote by $\Omega_{r}$ the infinite strip obtained from $\Omega_{\epsilon, r}$ as $\epsilon \rightarrow 0$, then for every $s \in \Omega_{r}$ we have

$$
\zeta_{A, \Lambda}(s)=\frac{1}{2 \pi i} \int_{\partial \Omega_{r}} \frac{\zeta_{A, \Lambda}(u) \mathrm{d} u}{u-s}
$$

Proof: Let us take $\epsilon^{\prime}<\epsilon$. Then the pre-image of the circle $|z-1|=\epsilon^{\prime}$ intersected with $\Omega$ is formed with two arcs $\eta_{3}^{\prime}$ inside $\eta_{3}$ and $\eta_{6}^{\prime}$ at the right of $\eta_{6}$. The arcs $\eta_{6}, \eta_{6}^{\prime}$ and the curves $\Gamma_{k, j}$ and $\Gamma_{k+1}^{\prime}$ determine a 
curvilinear quadrilateral whose conformal module is the same as that of the quadrilateral determined by $\gamma_{6}, \gamma_{6}^{\prime}$, the real axis and the segment from $z=1$ to $Z=\zeta_{A, \Lambda}\left(s_{1}\right)$, which in turn is less than the conformal module of the ring domain $\epsilon^{\prime} \leq|z-1| \leq \epsilon$. It is known (see [17], page 31) that the value of this last module is $\log \frac{\epsilon}{\epsilon^{\prime}}$. If we take $\epsilon^{\prime}=\frac{\epsilon}{2}$ then this module is $\log 2$, which shows that the length of $\eta_{6}$ remains bounded as $\epsilon \rightarrow 0$, since otherwise the respective module would tend to $\infty$, contrary to the fact that it remains constant $\log 2$. Let us evaluate $\int_{\eta_{6}} \frac{\zeta_{A, \Lambda}(u) \mathrm{d} u}{u-s}$. Since $\lim _{\mathrm{Re} u \rightarrow+\infty} \zeta_{\mathrm{A}, \Lambda}(u)=1$ we have that $\lim _{\mathrm{Re} u \rightarrow+\infty} \frac{\zeta_{A, \Lambda}(u)}{u-s}=0$ and since the length of $\eta_{6}$ remains bounded we have $\lim _{\mathrm{Re} u \rightarrow+\infty} \int_{\eta_{6}} \frac{\zeta_{\mathrm{A}, \Lambda}(u) \mathrm{d} u}{u-s}=0$.

On the other hand, by Cauchy theorem $\int_{\partial \Omega_{\epsilon, r}} \frac{\zeta_{A, \Lambda}(u) \mathrm{d} u}{u-S}$ does not depend on $\epsilon$ since $\int_{\partial \Omega_{\epsilon, r} \mid \partial \Omega_{\epsilon, r}} \frac{\zeta_{A, \Lambda}(u) \mathrm{d} u}{u-s}=0$. Then we can let $\epsilon \rightarrow 0$ in $\zeta_{A, \Lambda}(s)=\frac{1}{2 \pi i} \int_{\partial \Omega_{\epsilon, r}} \frac{\zeta_{A, \Lambda}(u) \mathrm{d} u}{u-s}$ and we obtain (3).

It is not clear what happens with $\int_{\eta_{1}} \frac{\zeta_{A, \Lambda}(u) \mathrm{d} u}{u-s}$ as $r \rightarrow \infty$. Making the change of variable $u=\varphi\left(r \mathrm{e}^{\mathrm{i} \theta}\right)$, where $\zeta_{A, \Lambda}\left(\varphi\left(r \mathrm{e}^{\mathrm{i} \theta}\right)\right)=r \mathrm{e}^{\mathrm{i} \theta}$, which is allowed since $\zeta_{A, \Lambda}(u)$ is injective on $\eta_{1}$, we get

$$
\int_{\eta_{1}} \frac{\zeta_{A, \Lambda}(u) \mathrm{d} u}{u-s}=\int_{0}^{2 \pi} \frac{r \mathrm{e}^{\mathrm{i} \theta}}{\varphi\left(r \mathrm{e}^{\mathrm{i} \theta}\right)-s} \frac{i r \mathrm{e}^{\mathrm{i} \theta}}{\zeta_{A, \Lambda}^{\prime}\left(\varphi\left(r \mathrm{e}^{\mathrm{i} \theta}\right)\right)} \mathrm{d} \theta=i r^{2} \int_{0}^{2 \pi} \frac{\mathrm{e}^{2 i \theta}}{\varphi\left(r \mathrm{e}^{i \theta}\right)-s} \frac{\mathrm{d} \theta}{\zeta_{A, \Lambda}^{\prime}\left(\varphi\left(r \mathrm{e}^{i \theta}\right)\right)} .
$$

Although the integrand tends to zero as $r \rightarrow \infty$, a limitation of the initial integral is problematic, due to the factor $r^{2}$ in the last term. So, as long as we cannot make sure that $\int_{\eta_{1}} \frac{\zeta_{A, \Lambda}(u) \mathrm{d} u}{u-s}$ tends to zero as $r \rightarrow \infty$, the problem of extending the Cauchy integral formula to the whole fundamental domain remains unsolved.

Theorem 2. Let $S_{k}$ be an arbitrary strip of the function $\zeta_{A, \Lambda}(s)$ as defined in Section 2 and for $r$ big enough let $\eta(r)$ be the part of the pre-image by $\zeta_{A, \Lambda}(s)$ of the circle $|z|=r$ included in $S_{k}$. We denote by $S_{k}(r)$ the part of the strip $S_{k}$ bounded at the left by $\eta(r)$. Then for every $s \in S_{k}(r)$ we have:

$$
\zeta_{A, \Lambda}(s)=\frac{1}{2 \pi i} \int_{\partial S_{k}(r)} \frac{\zeta_{A, \Lambda}(u) \mathrm{d} u}{u-s}
$$

Proof: For an arbitrary $\epsilon>0$ and for the given $r$, let us build the domains $\Omega_{\epsilon, r}$ as in Theorem 1 corresponding to every fundamental domain $\Omega \subset S_{k}$. The sum of the corresponding arcs $\eta_{1}$ from these domains is $\eta(r)$ and the sum of the corresponding arcs $\eta_{6}$ is an arc $\eta(\epsilon)$ connecting $\Gamma_{k}^{\prime}$ and $\Gamma_{k+1}^{\prime}$. The arcs $\eta_{3}$ squeeze each one to the respective points $u_{0}$ when $\epsilon \rightarrow 0$, where 
$\zeta_{A, \Lambda}\left(u_{0}\right)=1$.

Since for each arc $\eta_{6}$ we have $\lim _{\mathrm{Re} u \rightarrow+\infty} \int_{\eta_{6}} \frac{\zeta_{A, \Lambda}(u) \mathrm{d} u}{u-s}=0$ it results that $\lim _{\mathrm{Re} u \rightarrow+\infty} \int_{\eta(\epsilon)} \frac{\zeta_{A, \Lambda}(u) \mathrm{d} u}{u-s}=0$. Denoting by $S_{k}(\epsilon, r)$ the domain bounded by $\Gamma_{k}^{\prime}, \Gamma_{k+1}^{\prime}, \eta(r), \eta(\epsilon)$ and the arcs $\eta_{3}$ we see that this domain becomes $S_{k}(r)$ when $\epsilon=0$. On the other hand, the Cauchy integral formula is applicable to $S_{k}(\epsilon, r)$, i.e. for every $s \in S_{k}(\epsilon, r)$ we have $\zeta_{A, \Lambda}(s)=\frac{1}{2 \pi i} \int_{\partial S_{k}(\epsilon, r)} \frac{\zeta_{A, \Lambda}(u) \mathrm{d} u}{u-s}$ and then at the limit as $\epsilon \rightarrow 0$ this equality becomes (4) and the theorem is proved.

We notice that this theorem says that the values of $\zeta_{A, \Lambda}(s)$ are completely determined by its values greater than 1 and those taken on an arbitrary circle of radius big enough. Moreover, the integral of the formula (4) is always convergent.

If $s=s_{k}(x), x \geq 1$ is the parametric equation of $\Gamma_{k}^{\prime}$ such that $\zeta_{\mathrm{A}, \Lambda}\left(s_{k}(x)\right)=x$ then the formula (4) becomes

$$
\zeta_{A, \Lambda}(s)=\frac{1}{2 \pi i} \int_{1}^{r} x\left(\frac{s_{k+1}^{\prime}(x)}{s_{k+1}(x)-s_{0}}-\frac{s_{k}^{\prime}(x)}{s_{k}(x)-s_{0}}\right) \mathrm{d} x+\int_{\eta(r)} \frac{\zeta_{A, \Lambda}(u) \mathrm{d} u}{u-s}
$$

We notice that $\lim _{x \unlhd 1} s_{k}(x)=\infty$, hence the first integral in (5) is an improper integral. By Theorem 1 this integral is always convergent.

The function $\zeta_{A, \Lambda}(s)$ is not injective in $S_{k}$, hence the integrals (4) and (5) give us the same value for different points $s$ in $S_{k}$. If we would like to have a unique point corresponding to a given value, then we need to use the formula (3).

Also, taking into account the fact that the domain interior to every curve $\Gamma_{k, j}$, which is not embracing curve, is mapped conformally and therefore injectively by $\zeta_{A, \Lambda}(s)$ onto the upper or the lower half plane, a unique point $s$ from that domain corresponds to every given value from the respective half plane. Then the following formula is true for every $r$ big enough:

$$
\zeta_{A, \Lambda}(s)=\frac{1}{2 \pi i} \int_{\Gamma_{k, j}(r)} \frac{\zeta_{A, \Lambda}(u) \mathrm{d} u}{u-s}
$$

where $\Gamma_{k, j}(r)$ is the boundary of the domain bounded by $\Gamma_{k, j}$ and the preimage of the circle $|z|=r$.

If the equation of the curve $\Gamma_{k, j}$ is $s=s_{k, j}(x), x \in \mathbb{R}$ such that $\zeta_{A, \Lambda}\left(s_{k, j}(x)\right)=x$ then the formula (6) becomes

$$
\zeta_{A, \Lambda}(s)=\frac{1}{2 \pi i} \int_{-r}^{r} \frac{x s_{k, j}^{\prime}(x) \mathrm{d} x}{s_{k, j}(x)-s}+\frac{r^{2}}{2 \pi} \int_{0}^{2 \pi} \frac{\mathrm{e}^{2 i \theta}}{\varphi\left(r \mathrm{e}^{i \theta}\right)-s} \frac{\mathrm{d} \theta}{\zeta_{A, \Lambda}^{\prime}\left(\varphi\left(r \mathrm{e}^{i \theta}\right)\right)}
$$

where $\zeta_{A, \Lambda}\left(\varphi\left(r \mathrm{e}^{\mathrm{i} \theta}\right)\right)=r \mathrm{e}^{\mathrm{i} \theta}, 0 \leq \theta<2 \pi$.

\section{The Distribution of the Values of a Dirichlet Function}

The contour of integration in Theorem 2 is simpler than that appearing in Theorem 1. However, (3) has the advantage of representing a univalent function 
in $\Omega_{k, j}$. The pre-image of the circle $|z|=r$ intersects several components of the pre-image of the interval $(1,+\infty)$ of the real axis situated in $S_{k}$, more exactly if $\zeta_{A, \Lambda}(s)$ has $m$ zeros in $S_{k}$, then this pre-image intersects exactly $m+1$ such components, hence it traverses $m$ fundamental domains. Each one of these domains contains a unique point $s^{(j)}, j=1,2, \cdots, m$ such that $\zeta_{A, \Lambda}\left(s^{(j)}\right)=\zeta_{A, \Lambda}(s)$. A point on the circle $|z|=r$ should turn $m$ times around the origin for its pre-image to traverse the $m$ fundamental domains going from $\Gamma_{k+1}^{\prime}$ to $\Gamma_{k}^{\prime}$. At every turn it assigns a unique point $s^{(j)}$ where $\zeta_{A, \Lambda}(s)$ takes the same value. Since $\zeta_{A, \Lambda}(s)$ is univalent in $\Omega_{k, j}$ that value is completely determined by the values on $\Gamma_{k+1}^{\prime}$ and $\Gamma_{k}^{\prime}$.

\section{Extension of Cauchy Integral Formula for the Derivatives of Dirichlet Functions}

Following the known technique of computing $\lim _{h \rightarrow 0} \frac{1}{h}\left[\zeta_{A, \Lambda}\left(s_{0}+h\right)-\zeta_{A, \Lambda}\left(s_{0}\right)\right]$ we find that

$$
\zeta_{A, \Lambda}^{\prime}\left(s_{0}\right)=\frac{1}{2 \pi i} \int_{\partial S_{k}(r)} \frac{\zeta_{A, \Lambda}(s) \mathrm{d} s}{\left(s-s_{0}\right)^{2}}
$$

Thus, as for $\zeta_{A, \Lambda}(s)$ the values of $\zeta_{A, \Lambda}^{\prime}(s)$ are completely determined in every strip $S_{k}$ by the real values greater than 1 of $\zeta_{A, \Lambda}(s)$. By recursive computation we find that:

$$
\zeta_{A, \Lambda}^{(n)}\left(s_{0}\right)=\frac{n !}{2 \pi i} \int_{\partial S_{k}} \frac{\zeta_{A, \Lambda}(s) \mathrm{d} s}{\left(s-s_{0}\right)^{n+1}}
$$

for every natural number $n$.

It is known (see [4] and Figure 4) that if a Dirichlet L-function $L(\chi ; s)$ has $m$ zeros in the strip $S_{k}$ then $L^{(n)}(\chi ; s), n \geq 1$ has $m-1$ zeros in $S_{k}$ (which are all simple zeros).

This figure illustrates the following:

Theorem 3. If $\zeta_{A, \Lambda}(s)$ has $m$ fundamental domains in $S_{k}$ then every derivative $\zeta_{A, \Lambda}^{(n)}(s)$ has exactly $m-1$ zeros in $S_{k}$.

Proof: The pre-image by $\zeta_{A, \Lambda}(s)$ of a circle $|z|=r$ has $m$ components in $S_{k}$ which are disjoint if $r$ is small enough. By letting $r$ increase these components expand and after two of them touch each other, they fuse into a unique component including the corresponding zeros. When $r=1$ a unique component becomes unbounded with branches tending to $\infty$ with $\operatorname{Re} s \rightarrow+\infty$. The remaining bounded components are outside this unbounded one and none of them can intersect $\partial S_{k}$. Increasing $r$ past 1 the unbounded components from all the strips $S_{k}$ fuse into a unique unbounded component crossing these strips. After touching bounded components of the pre-image of the circle $|z|=r$ with $r>1$ (if such components exist in a given $S_{k}$ ), these last components are absorbed into the unbounded one and the corresponding zeros of $\zeta_{A, \Lambda}(s)$ pass 


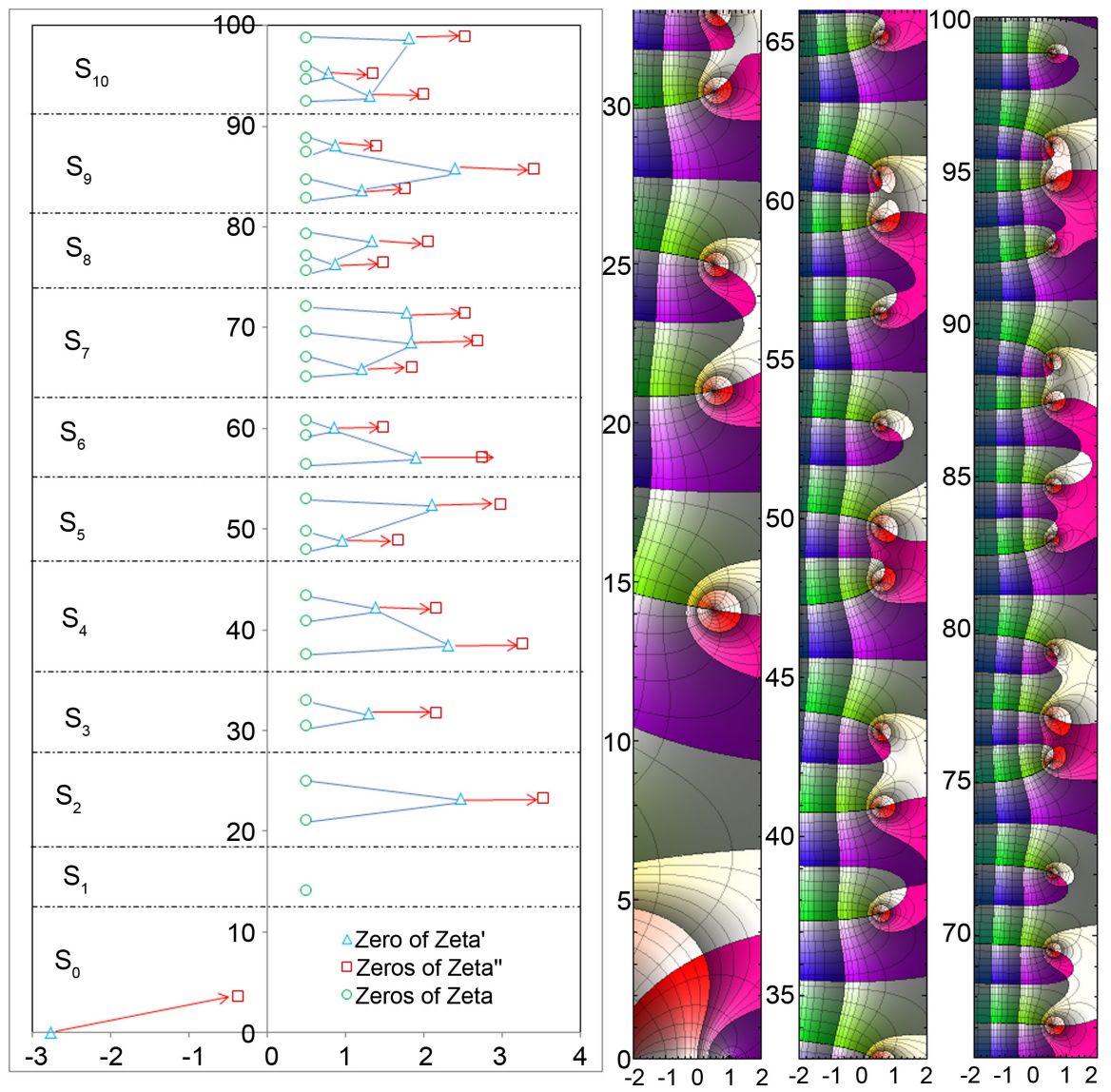

Figure 4. The zeros of $\zeta(s)$ and those of its first two derivatives for $t \in(0,100)$.

to the right of it. For $r$ big enough all the zeros of $\zeta_{A, \Lambda}(s)$ from the strip $S_{k}$ will be at the right of the unbounded component of the pre-image of the circle $|z|=r$. That value of $r$ depends on $S_{k}$.

The points where two components of the pre-image of a circle $|z|=r$ touch each other are the zeros of $\zeta_{A, \Lambda}^{\prime}(s)$. A complete binary tree can be formed having as leaves the zeros of $\zeta_{A, \Lambda}(s)$ and as internal nodes these touching points. It is known from the graph theory that if a complete binary tree has $m$ leaves then it has exactly $m-1$ internal nodes. This proves that $\zeta_{A, \Lambda}^{\prime}(s)$ has $m-1$ zeros in $S_{k}$. The zeros of the second derivative are obtained in a similar way, yet since $\lim _{\sigma \rightarrow+\infty} \zeta_{A, \Lambda}^{\prime}(\sigma+i t)=0$, there will be $m$ components of the pre-image by $\zeta_{A, \Lambda}^{\prime}(s)$ of the circle $|z|=r$ for $r$ small enough, even if there are only m-1 zeros of $\zeta_{A, \Lambda}^{\prime}(s)$. One of these components contains no such a zero. These components touch each other at the zeros of $\zeta_{A, \Lambda}^{\prime \prime}(s)$ and by the previous analysis there should be $m-1$ zeros of $\zeta_{A, \Lambda}^{\prime \prime}(s)$. The same procedure can be applied to derivatives of any higher order and the theorem is completely proved.

Figure 5 portraying the pre-image of the real axis by the Riemann Zeta function and by its derivative shows that their $S_{k}$-strips and their fundamental domains overlap, but they do not completely coincide (see [11]). However, the 

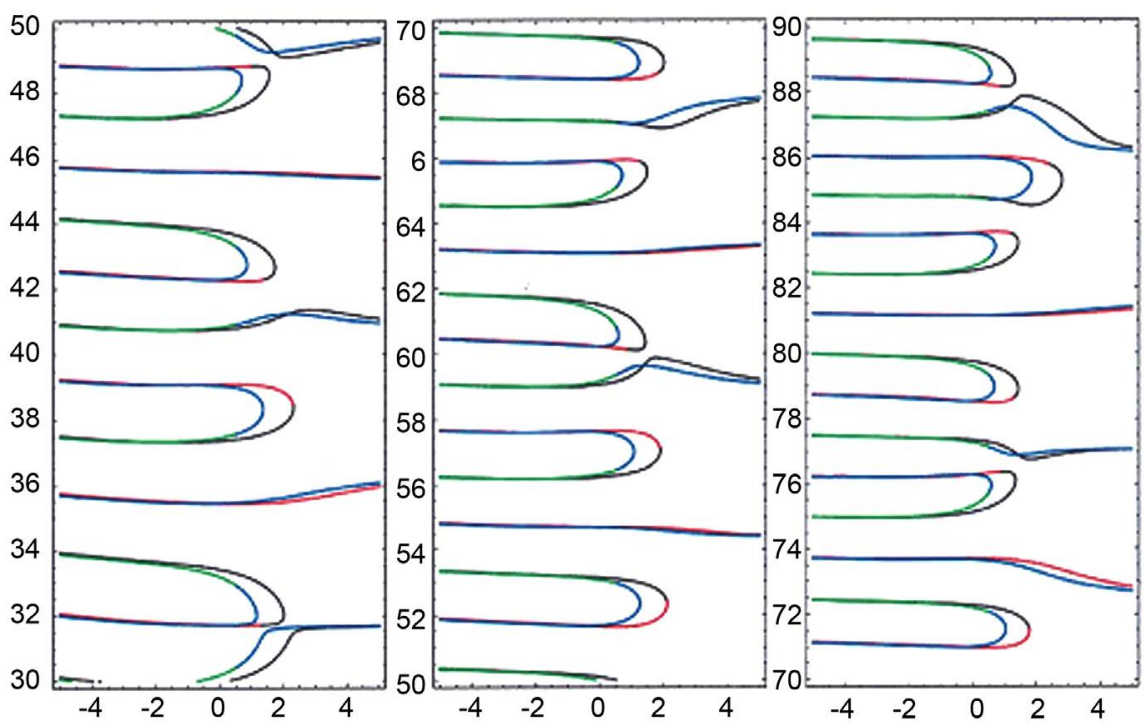

Figure 5. The pre-image of the real axis by $\zeta(s)$ and $\zeta^{\prime}(s)$.

integral (8) gives the same value for $\zeta_{A, \Lambda}^{\prime}\left(s_{0}\right)$ as

$$
\zeta_{A, \Lambda}^{\prime}\left(s_{0}\right)=\frac{1}{2 \pi i} \int_{\partial S_{k}(r)} \frac{\zeta_{A, \Lambda}^{\prime}(s) \mathrm{d} s}{s-s_{0}}
$$

since if $\Sigma_{k}$ is the corresponding strip of $\zeta_{A, \Lambda}^{\prime}(s)$ the integral on $\partial\left(S_{k}(r) \cap \Sigma_{k}(r)\right)$ is zero, by Cauchy Theorem. The same is true for the integrals on the boundaries of the corresponding fundamental domains of the two functions.

\section{Extension of Cauchy Integral Formula to Fundamental Domains of Modular Function}

By the Riemann mapping theorem there is a unique analytic function $z=\lambda(s)$ mapping conformally the domain $D$ bounded by the half lines $\operatorname{Re} s=0$, $\operatorname{Re} s=1, \operatorname{Im} s \geq 0$ and the half circle $s=\frac{1}{2}\left(1+\mathrm{e}^{i \theta}\right), 0 \leq \theta \leq \pi$ onto the upper half plane $\operatorname{Re} z>0$ such that $\lambda(0)=1, \lambda(1)=\infty$ and $\lambda(\infty)=0$. The function $\lambda(s)$ can be continued by symmetry into the upper half plane as in Figure 6.

The symmetric domain $D^{\prime}$ of $D$ with respect to the imaginary axis is mapped conformally by $\lambda(s)$ onto the lower half plane and $\Omega=D \cup D^{\prime} \bigcup\{s \mid \operatorname{Re} s=0, \operatorname{Im} s \geq 0\} \quad$ is mapped conformally onto the whole complex plane with the slit $(-\infty, 0] \cup[1,+\infty)$, alongside the real axis, hence $\Omega$ is a fundamental domain of $\lambda(s)$. In fact the union any two adjacent domains bounded by the half circles above (the half lines can be considered half circles too!) and the common half circle is a fundamental domain of $\lambda(s)$. For example $D_{1} \cup D_{2} \cup\{s|| s-1 / 2 \mid=1 / 2, \operatorname{Im} s \geq 0\}$ is mapped conformally by $\lambda(s)$ onto the whole complex plane with the slit $(-\infty, 1]$ and $D_{1} \cup D_{2} \cup\{s|| s-1 / 4 \mid=1 / 4, \operatorname{Im} s \geq 0\}$ is mapped conformally by $\lambda(s)$ onto the 


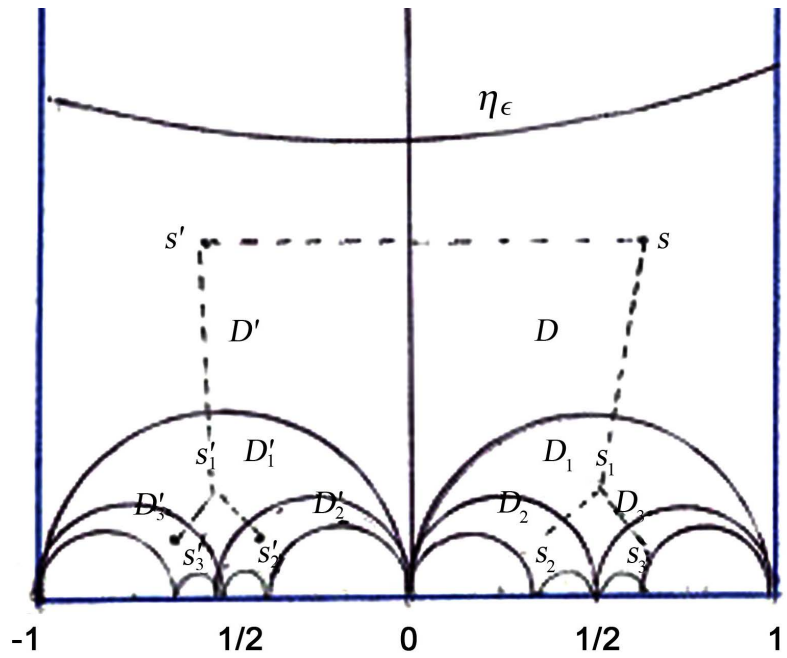

Figure 6. Continuation by symmetry of the modular function.

whole complex plane with the slit $[0,+\infty)$ etc. So, Figure 6 exhibits a partition of the upper half plane into fundamental domains of $\lambda(s)$. Each one of these half circles ends up into a singular point of $\lambda(s)$, therefore these singular points form a dense subset of the real axis, which implies that $\lambda(s)$ cannot be continued across the real axis and its full domain of definition is the upper half plane.

The way the function $\lambda(s)$ has been constructed implies that for every $s$ in the upper half plane we have $\lambda(s+2)=\lambda(s)$ and $\lambda\left(\frac{s}{2 s+1}\right)=\lambda(s)$ and then (see [1], page 280) $\lim _{t \rightarrow+\infty} \lambda(\sigma+i t)=0$ uniformly with respect to $\sigma$. Hence the part of the pre-image by $\lambda(s)$ of the circle $z=\epsilon \mathrm{e}^{\mathrm{i} \theta}, 0 \leq \theta<2 \pi$ included in $\Omega$ is an $\operatorname{arc} \eta_{\epsilon}: s=\sigma\left(\epsilon \mathrm{e}^{i \theta}\right)+i t\left(\epsilon \mathrm{e}^{i \theta}\right), \quad 0 \leq \theta<2 \pi$, where $\lim _{\epsilon \rightarrow 0} t\left(\epsilon \mathrm{e}^{i \theta}\right)=\infty$ uniformly with respect to $\theta$. In particular, denoting by $v(z)$ the inverse of $\lambda(s)$ in $\Omega$, we have $\int_{\eta_{\epsilon}} \frac{\lambda(s)}{s-s_{0}} \mathrm{~d} s=\int_{0}^{2 \pi} \frac{\epsilon \mathrm{e}^{\mathrm{i} \theta}}{v\left(\epsilon \mathrm{e}^{i \theta}\right)-s_{0}} \frac{i \epsilon \mathrm{e}^{i \theta} \mathrm{d} \theta}{\lambda^{\prime}\left(v\left(\epsilon \mathrm{e}^{\mathrm{i} \theta}\right)\right)} \rightarrow 0 \quad$ as $\epsilon \rightarrow 0$.

On the other hand, the part of the pre-image by $\lambda(s)$ of the circle $z=r e^{i \theta}, 0 \leq \theta<2 \pi$ included in $\Omega$ is formed for $r$ big enough with two arcs $\eta_{r, 1}$ and $\eta_{r,-1}$ such that for $s \in \eta_{r, 1}$ we have $s \rightarrow 1$ as $r \rightarrow \infty$ and for $s \in \eta_{r,-1}$ we have $s \rightarrow-1$ as $r \rightarrow \infty$. Let us denote by $\Omega_{r}$ the part of $\Omega$ obtained by removing the pre-image of the set $\{z|| z \mid \geq r\}$.

Theorem 4. For any fundamental domain $\Omega$ of the function $\lambda(s)$ and any point $s_{0} \in \Omega_{r}$ we have:

$$
\lambda\left(s_{0}\right)=\int_{\partial \Omega_{r}} \frac{\lambda(s)}{s-s_{0}} \mathrm{~d} s
$$

Proof: Let us deal first with the fundamental domain $\Omega=D \cup D^{\prime} \bigcup\{s \mid \operatorname{Re} s=0, \operatorname{Im} s \geq 0\}$. By isolating the point $z=0$ with a small circle $Z=\epsilon \mathrm{e}^{\mathrm{i} \theta}, 0 \leq \theta<2 \pi$ and the point $Z=\infty$ with a big circle $z=r e^{i \theta}, 0 \leq \theta<2 \pi$ and by removing from $\Omega$ the pre-image of the disc $|z| \leq \epsilon$ 
and of the exterior of the disc $|z|<r$ we obtain a bounded domain $\Omega_{\epsilon, r}$ for which the Cauchy integral formula is applicable: for any $s_{0} \in \Omega_{\epsilon, r}$ we have $\lambda\left(s_{0}\right)=\int_{\partial \Omega_{\epsilon, r}} \frac{\lambda(s)}{s-s_{0}} \mathrm{~d} s$. Taking $\epsilon^{\prime}<\epsilon$ and having in view the Cauchy theorem we conclude that $\int_{\partial \Omega_{\epsilon, r}} \frac{\lambda(s)}{s-s_{0}} \mathrm{~d} s=\int_{\partial \Omega_{\epsilon}, r} \frac{\lambda(s)}{s-s_{0}} \mathrm{~d} s$ and since $\lim _{\epsilon \rightarrow 0} \int_{\eta_{\epsilon}} \frac{\lambda(s)}{S-s_{0}} \mathrm{~d} s=0$, we can set $\epsilon=0$ and get (11).

Now, if we take for example $\Omega=D \cup D_{1} \cup\{s|| s-1 / 2 \mid=1 / 2, \operatorname{Im} s \geq 0\}$ and proceed similarly, the new $\eta_{\epsilon}$ is the part of the pre-image of the circle $|z|=\epsilon$ included in $D$, hence again.

$\lim _{\epsilon \rightarrow 0} \int_{\eta_{\epsilon}} \frac{\lambda(s)}{s-s_{0}} \mathrm{~d} s=0$ and the extension of the Cauchy integral formula is true for the new unbounded domain $\Omega_{r}$. We are brought to the same conclusion when $\Omega=D \cup D_{1}^{\prime} \bigcup\{s|| s+1 / 2 \mid=1 / 2, \operatorname{Im} s \geq 0\}$.

This theorem tells us that the modular function is completely determined by its real values and by the values on the pre-image of an arbitrary big circle centered at the origin.

\section{Extension of Cauchy Integral Formula to the Fundamental Domains of the Exponential Function}

It is known that the horizontal strips bounded by consecutive lines $\operatorname{Im} z=2 k \pi$ are fundamental domains $\Omega_{k}$ of the exponential function $w=\mathrm{e}^{z}$. The function $\mathrm{e}^{z}$ maps conformally each one of these strips onto the complex plane with the slit alongside the positive real half axis. The pre-image by $\mathrm{e}^{z}$ of the circle $|w|=r$ is the vertical line $\operatorname{Re} z=\ln r$ and if we denote by $\gamma$ the intersection of this line with any fundamental domain of $\mathrm{e}^{z}$ then $\int_{\gamma} \frac{\mathrm{e}^{z}}{z-z_{0}} \mathrm{~d} z=\int_{2 k \pi}^{2(k+1) \pi} \frac{r \mathrm{e}^{\mathrm{i} \theta}}{\ln r+i \theta-z_{0}} i \mathrm{~d} \theta=\frac{i r}{\ln r} \int_{2 k \pi}^{2(k+1) \pi} \frac{\mathrm{e}^{\mathrm{i} \theta} \mathrm{d} \theta}{1+\frac{i \theta-z_{0}}{\ln r}}$ and at the limit as $r \rightarrow \infty$ we get an indetermination of the form $\infty * 0$. Thus $\int_{\partial \Omega_{k}} \frac{\mathrm{e}^{z}}{z-z_{0}} \mathrm{~d} z$ might be divergent. However, we can prove:

Theorem 5. For any fundamental domain $\Omega_{k}$ of $\mathrm{e}^{z}$ and for any positive number $r$, if we denote $\Omega_{k}(r)=\Omega_{k} \cap\{z \mid \operatorname{Re} z \leq \ln r\}$ we have

$$
\mathrm{e}^{z_{0}}=\frac{1}{2 \pi i} \int_{\partial \Omega_{k}(r)} \frac{\mathrm{e}^{z}}{z-z_{0}} \mathrm{~d} z
$$

where $z_{0}$ is an arbitrary point of $\Omega_{k}(r)$.

Proof: The intersection of the pre-image by $\mathrm{e}^{z}$ of the annulus $\rho \leq|w| \leq r$ and $\Omega_{k}$ is a bounded domain $D_{k}(\rho, r)$ and for every $z_{0} \in D_{k}(\rho, r)$ we have

$$
\mathrm{e}^{z_{0}}=\frac{1}{2 \pi i} \int_{\partial D_{k}(\rho, r)} \frac{\mathrm{e}^{z}}{z-z_{0}} \mathrm{~d} z
$$


In order to obtain (12) it will be enough to show that $\lim _{\rho \rightarrow 0} \int_{\gamma_{\rho}} \frac{\mathrm{e}^{z}}{z-z_{0}} \mathrm{dz}=0$, where $\gamma_{\rho}$ is $\Omega_{k} \cap\left\{z=x+i y \mid \mathrm{e}^{x}=\rho\right\}$. This integral is $\int_{2 k \pi}^{2(k+1) \pi} \frac{\rho \mathrm{e}^{\mathrm{i} \theta}}{\ln \rho+i \theta-z_{0}} i \mathrm{~d} \theta=\frac{i \rho}{\ln \rho} \int_{2 k \pi}^{2(k+1) \pi} \frac{\mathrm{e}^{\mathrm{i} \theta} \mathrm{d} \theta}{1+\frac{i \theta-z_{0}}{\ln \rho}}$ and since the integrand is bounded and $\lim _{\rho \rightarrow 0} \frac{\rho}{\ln \rho}=0$ we obtain at the limit the formula (12).

\section{The Case of Trigonometric Functions}

We illustrate this case by dealing with the function $w=\cos z \quad$ (see [9], page 51). for which the fundamental domains are vertical half strips $\Omega_{j}, j \in \mathbb{Z}$ determined by the lines $\operatorname{Re} z=2 j \pi, \operatorname{Im} z \geq 0$ and $\bar{\Omega}_{j}$, symmetric of $\Omega_{j}$ with respect to the real axis. They are mapped conformally by the function $\cos z$ onto the complex plane with a slit alongside the interval $[-1,+\infty)$. For $r>1$, the pre-image of the circle $w=r \mathrm{e}^{i \theta}$ is the curve of equation $\cos (x+i y)=r(\cos \theta+i \sin \theta)$. An elementary computation shows that this equation is equivalent to shy $= \pm \sqrt{r^{2}-\cos ^{2} x}$. This show that $\sqrt{r^{2}-1} \leq$ shy $\leq r$ in $\Omega_{j}$ and $-r \leq$ shy $\leq-\sqrt{r^{2}-1}$ in $\bar{\Omega}_{j}$ and shy $= \pm r$ when $x=(2 k+1)(\pi / 2)$, respectively shy $= \pm \sqrt{r^{2}-1}$ when $x=k \pi$. Hence the pre-image of the circle $w=r \mathrm{e}^{i \theta}$ is formed with two sinusoidal curves symmetric with respect to the real axis. Moreover, $\lim _{r \rightarrow \infty} \frac{s h y}{r}=1$ in $\Omega_{j}$ and $\lim _{r \rightarrow \infty} \frac{s h y}{r}=-1$ in $\bar{\Omega}_{j}$ uniformly with respect to $x$ when $x+i y$ is on either one of these curves. Let us denote by $\gamma_{j}$ the part of this pre-image situated in $\Omega_{j}$. We would like to evaluate $\int_{\gamma_{j}} \frac{\cos Z}{Z-Z_{0}} \mathrm{~d} z$. For every domain $\Omega_{j}$ there is an analytic function $\varphi(w)$ such that $\cos \left(\varphi\left(r \mathrm{e}^{\mathrm{i} \theta}\right)\right)=r \mathrm{e}^{\mathrm{i} \theta}$. Then making the change of variable $z=\varphi\left(r \mathrm{e}^{i \theta}\right), 2 j \pi \leq \theta \leq 2(j+1) \pi$ in this integral we get

$$
\begin{aligned}
\int_{\gamma_{j}} \frac{\cos z}{Z-z_{0}} \mathrm{~d} z & =\int_{2 j \pi}^{2(j+1) \pi} \frac{r \mathrm{e}^{\mathrm{i} \theta}}{\varphi\left(r \mathrm{e}^{\mathrm{i} \theta}\right)-z_{0}} \frac{-i r \mathrm{e}^{\mathrm{i} \theta}}{\sin \left(\varphi\left(r \mathrm{e}^{\mathrm{i} \theta}\right)\right)} \mathrm{d} \theta \\
& =-i r^{2} \int_{2 j \pi}^{2(j+1) \pi} \frac{\mathrm{e}^{2 i \theta}}{\varphi\left(r \mathrm{e}^{\mathrm{i} \theta}\right)-z_{0}} \frac{1}{\sin \left(\varphi\left(r \mathrm{e}^{\mathrm{i} \theta}\right)\right)} \mathrm{d} \theta
\end{aligned} .
$$

Let us notice that $\sin \left(\varphi\left(r \mathrm{e}^{-i \theta}\right)\right)= \pm \sqrt{1-\cos ^{2}\left(\varphi\left(r \mathrm{e}^{-i \theta}\right)\right)}= \pm \sqrt{1-r^{2} \mathrm{e}^{-2 i \theta}}$ and we need to chose the sine minus in the last term since if to $0 \leq \theta<\pi$ corresponds $x=\operatorname{Re} \varphi\left(r e^{-i \theta}\right)>0$ then to $-\theta$ corresponds $x<0$, hence $\sin \left(\varphi\left(r \mathrm{e}^{i \theta}\right)\right)$ is an odd function of $\theta$.

By making the change of variable $\theta \rightarrow \theta+2 j \pi$, we get

$$
\begin{aligned}
\int_{\gamma_{j}} \frac{\cos z}{z-z_{0}} \mathrm{~d} z & =i r^{2} \int_{0}^{2 \pi} \frac{\mathrm{e}^{2 i \theta}}{\varphi\left(r \mathrm{e}^{i \theta}\right)-z_{0}} \frac{-1}{\sin \left(\varphi\left(r \mathrm{e}^{\mathrm{i} \theta}\right)\right)} \mathrm{d} \theta \\
& =i r^{2} \int_{-\pi}^{\pi} \frac{\mathrm{e}^{2 i \theta}}{\varphi\left(r \mathrm{e}^{\mathrm{i} \theta}\right)-z_{0}} \frac{-1}{\sin \left(\varphi\left(r \mathrm{e}^{\mathrm{i} \theta}\right)\right)} \mathrm{d} \theta
\end{aligned}
$$


When $r$ is big enough the term $\varphi\left(r \mathrm{e}^{\mathrm{i} \theta}\right)-z_{0}$ varies very little as $\theta$ varies from $-\pi$ to $\pi$. Assuming a constant instead of this term, what it remains to integrate between $-\pi$ and $\pi$ is an odd function, hence the integral is zero. This doesn't necessarily mean that $\lim _{r \rightarrow \infty} \int_{\gamma_{j}} \frac{\cos z}{z-z_{0}} d z=0$. However, the previous remark justifies the conjecture that this limit is true and therefore the Cauchy integral formula extends to the boundaries of the fundamental domains of the function $\cos z$.

\section{Extension of Cauchy Integral Formula to the Fundamental Domains of the Weierstrass $\wp$ Function}

The Weierstrass $\wp$ function is defined (see [1], page 272) by the formula

$$
\wp(z)=\frac{1}{z^{2}}+\sum_{\omega \neq 0}\left(\frac{1}{(z-\omega)^{2}}-\frac{1}{\omega^{2}}\right)
$$

where the sum ranges over all $\omega=m \omega_{1}+n \omega_{2}$, where $(m, n) \in \mathbb{Z} \times \mathbb{Z} \backslash(0,0)$ with $\omega_{1}$ and $\omega_{2}$ arbitrary complex numbers having non real ratio $\omega_{2} / \omega_{1}$. It is known that $w=\wp(z)$ is a doubly periodic function with the periods $\omega_{1}$ and $\omega_{2}$. Hence it is sufficient to know the values of $\wp(z)$ into the (fundamental) parallelogram determined by $\omega_{1}$ and $\omega_{2}$ in order to be able to find its values anywhere in the complex plane. The series (15) converges uniformly and absolutely on any compact subset of $\mathbb{C}$ which does not contain points $\omega$, therefore it is a meromorphic function in the complex plane. The points $\omega$ are double poles for $\wp(z)$ and hence they are triple poles for $\wp^{\prime}(z)=-2 \sum_{\omega \neq 0} \frac{1}{(z-\omega)^{3}}$.

It can be easily shown that $\wp^{\prime}\left(\omega_{1} / 2\right)=\wp^{\prime}\left(\omega_{2} / 2\right)=\wp^{\prime}\left(\omega_{1} / 2+\omega_{2} / 2\right)=0$. Moreover, since $\wp(z)=\wp(-z)=\wp\left(\omega_{1}+\omega_{2}-z\right)$, by denoting $z^{\prime}=\omega_{1}+\omega_{2}-z$, we have $\frac{z+z^{\prime}}{2}=\frac{\omega_{1}+\omega_{2}}{2}$, thus $z$ and $z^{\prime}$ are symmetric with respect to the center of the fundamental parallelogram, hence if we know the values of $\wp(z)$ in one of the triangles determined by a diagonal of the parallelogram, then we know its values in the whole parallelogram. Also, $\wp(z)$ takes the same value at points symmetric with respect to the middle of each one of the sides of this triangle. Since the function is univalent in the triangle and maps each side two to one onto some curve originating in the image of the middle of the respective side and going to infinity (the ends of each side being poles) we conclude that these triangles are fundamental domains of $\wp(z)$. Let us denote $e_{1}=\wp\left(\omega_{1} / 2\right)$, $e_{2}=\wp\left(\omega_{2} / 2\right)$ and $e_{3}=\wp\left(\omega_{1} / 2+\omega_{2} / 2\right)$. Then $\wp(z)$ maps conformally every fundamental triangle onto the whole complex plane with infinite slits originating at $e_{1}, e_{2}$ and $e_{3}$. Figure 7 illustrates this situation. It shows also that each one of the domains $D_{k}, k=0,1,2,3$ is mapped conformally onto the corresponding domain $D_{k}^{\prime}$ with two sides of $D_{k}, k=0,1,2,3$ going onto the slits. From the 


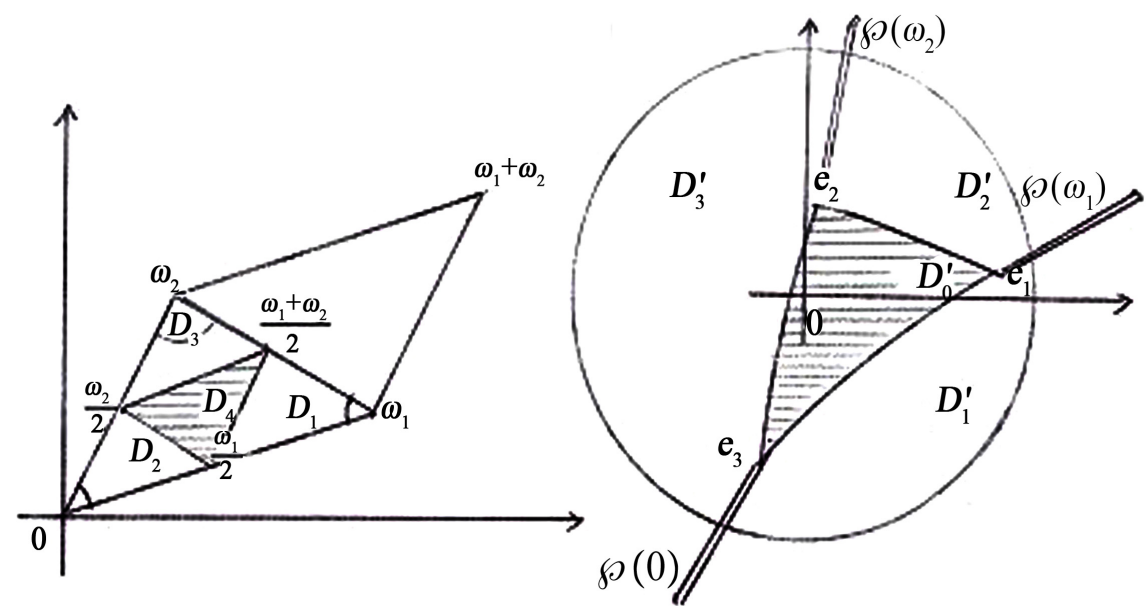

Figure 7. Fundamental triangle of $\wp(z)$ and its conformal mapping.

differential equation of $\wp(z)$ (see [1], page 278) is obvious that $e_{1}+e_{2}+e_{3}=0$, which implies that the triangle with vertices $e_{1}, e_{2}, e_{3}$ contains the origin, hence $\wp(z)$ has a zero in the domain $D_{0}$ and, obviously another one in the symmetric domain with respect to $\frac{\omega_{1}+\omega_{2}}{2}$ (These might have been unknown facts until now!).

Theorem 6. The Cauchy integral formula can be extended to any fundamental domain of the Weierstrass $\wp$ function .

Proof. For $r$ big enough the circle $|w|=r$ is intersecting every slit of $\wp(z)$ and the pre-image by $\wp(z)$ of the domain $\{w|| w \mid>r\}$ is formed with infinitely many connected open sets covering each one a vertex of the period parallelograms. The function $\wp(z)$ is analytic on the complementary set of this pre-image. In particular, the Cauchy integral formula is applicable to any fundamental triangle from which that pre-image has been removed. If we denote by $\Omega_{r}$ such a set, then we have:

$$
\wp\left(z_{0}\right)=\frac{1}{2 \pi i} \int_{\partial \Omega_{r}} \frac{\wp(z)}{Z-z_{0}} \mathrm{~d} z
$$

for every $z_{0} \in \Omega_{r}$. Due to the univalence of $\wp(z)$ in $\Omega_{r}$, it has an inverse function $\varphi(w)$ defined in the disc $|w| \leq r$. Thus, $\wp\left(\varphi\left(r \mathrm{e}^{\mathrm{i} \theta}\right)\right)=r \mathrm{e}^{\mathrm{i} \theta}$. With the change of variable $z=\varphi\left(r \mathrm{e}^{\mathrm{i} \theta}\right)$, the integral on the part of $\partial \Omega_{r}$ belonging to the pre-image of the circle $w=r \mathrm{e}^{\mathrm{i} \theta}$ becomes

$$
\int_{0}^{2 \pi} \frac{r \mathrm{e}^{\mathrm{i} \theta}}{\varphi\left(r \mathrm{e}^{i \theta}\right)-z_{0}} \frac{i r \mathrm{e}^{\mathrm{i} \theta} \mathrm{d} \theta}{\wp^{\prime}\left(\varphi\left(r \mathrm{e}^{\mathrm{i} \theta}\right)\right)}
$$

Since the points $\omega$ are triple poles $\wp^{\prime}(z)$ the term $\wp^{\prime}\left(\varphi\left(r \mathrm{e}^{\mathrm{i} \theta}\right)\right)$ tends to infinity as fast as $r^{3}$ when $r \rightarrow \infty$ therefore the integrand in (18) tends to zero as $r \rightarrow \infty$. If we denote by $\Omega$ any fundamental domain of $\wp(z)$, then this limit guarantees the absolute convergence of the improper integral $\int_{\partial \Omega} \frac{\wp(z)}{z-z_{0}} \mathrm{~d} z$ and 
the fact that for every $z_{0} \in \Omega$ we have:

$$
\wp\left(z_{0}\right)=\frac{1}{2 \pi i} \int_{\partial \Omega} \frac{\wp(z)}{z-z_{0}} \mathrm{~d} z
$$

which represents the extension of Cauchy integral formula to the fundamental domains of the Weierstrass $\wp$ function. It asserts that the function is completely determined by its values on the boundary of any fundamental triangle.

Theorem 7. For any fundamental domain $\Omega$ of $\wp(z)$ and for every point $z_{0} \in \Omega$ the value of an arbitrary derivative $\wp^{(k)}(z)$ at $z_{0}$ is given by the formula:

$$
\wp^{(k)}\left(z_{0}\right)=\frac{k !}{2 \pi i} \int_{\partial \Omega} \frac{\wp(z)}{\left(z-z_{0}\right)^{k+1}} \mathrm{~d} z=\frac{1}{2 \pi i} \int_{\partial \Omega} \frac{\wp^{(k)}(z)}{z-z_{0}} \mathrm{~d} z
$$

Proof: Since the integral (18) converges absolutely, we can differentiate term by term in (18) with respect to $z_{0}$ as many times as we want and we obtain the first equality in (19). For the second equality we write (17) with $\wp^{(k)}(z)$ instead $\wp(z)$ and notice that the corresponding term in (17) still tends to zero as $r \rightarrow \infty$. Then a formula similar to (18) is true with $\wp(z)$ replaced by $\wp^{(k)}(z)$, hence the improper integral $\int_{\partial \Omega} \frac{\wp^{(k)}(z)}{z-z_{0}} \mathrm{~d} z$ converges absolutely and we obtain the second equality in (19).

\section{An Integral Formula for the Weierstrass $\zeta$-Function}

Weierstrass denoted the antiderivative of $\wp(z)$ (which is defined up to an additive constant) by $-\zeta(z)$. Therefore $\zeta^{\prime}(z)=-\wp(z)$ and if we normalize it so that it is odd (see [1], page 273) we get $\zeta(z)=\frac{1}{Z}+\sum_{\omega \neq 0}\left(\frac{1}{z-\omega}+\frac{1}{\omega}+\frac{z}{\omega^{2}}\right)$. The series converges absolutely and uniformly on every compact set which does not contain any period point $\omega$. We obtain $\zeta(z)$ by integrating on any path that does not pass trough the poles the function $-\wp(s)$ from $z_{0}$ to $Z$, where we can take $z_{0}$ such that $\zeta^{\prime}\left(z_{0}\right)=0$. Then having in view (18) we can write:

$$
\zeta(z)=-\frac{1}{2 \pi i} \int_{z_{0}}^{z}\left(\int_{\partial \Omega} \frac{\zeta^{\prime}(s)}{s-u} \mathrm{~d} s\right) \mathrm{d} u
$$

\section{Conclusion}

The concept of fundamental domain, as defined by Ahlfors (see [1], page 99), is crucial in understanding the geometry of the mappings by analytic functions. We realized that the Cauchy integral formula can be extended to the boundary of such a domain. However, this extension cannot be performed for an arbitrary analytic function and the process requires specific treatment for specific classes of such functions. We selected in this paper classes of functions we thought to be the most representative. The selection is far from exhaustive and a lot of work remains to be done. 


\section{Acknowledgements}

We thank Aneta Costin for her support with technical matters.

\section{Conflicts of Interest}

The author declares no conflicts of interest regarding the publication of this paper.

\section{References}

[1] Ahlfors, L.V. (1953) Complex Analysis. McGraw-Hill, New York.

[2] Ballantine, C. and Ghisa, D. (2009) Global Mapping Properties of Rational Functions. Proceedings of 7 th ISAAC Congress, London, 13-18 July 2009, 13-22. https://doi.org/10.1142/9789814313179 0002

[3] Andreian Cazacu, C. and Ghisa, D. (2009) Global Mapping Properties of Analytic Functions. Proceedings of 7 th ISAAC Congress, London, 13-18 July 2009, 3-12. https://doi.org/10.1142/9789814313179 0001

[4] Barza, I., Ghisa, D. and Muscutar, F.A. (2014) On the Location of the Zeros of the Derivative of Dirichlet $L$-Functions. Annals of the University of Bucharest, 5, 21-31.

[5] Cao-Huu, T., Ghisa, D. and Muscutar, F.A. (2016) Multiple Solutions of Riemann Type of Functional Equations. British Journal of Mathematics \& Computer Science, 17, 1-12. https://doi.org/10.9734/BJMCS/2016/26322

[6] Cao-Huu, T., Ghisa, D. and Muscutar, F.A. (2019) Hidden Symmetries of Complex Analysis. Advances in Pure Mathematics, 9, 844-856.

[7] Ghisa, D. (2019) On the Zeros of Euler Product Dirichlet Functions. APM, 9, 959-966. https://doi.org/10.4236/apm.2019.912048

[8] Ghisa, D. (2018) Fundamental Domains of Dirichlet Functions. Nineteenth International Conference on Geometry, Integrability and Quantization, Varna, 1-30.

[9] Ghisa, D. (2012) Fundamental Domains and Riemann Hypothesis. Lambert Academic Publishing, Saarbrücken.

[10] Ghisa, D. (2014) On the Generalized Riemann Hypothesis, Complex Analysis and Potential Theory with Applications. Cambridge Scientific Publishers, Cambridge, 77-94.

[11] Ghisa, D. (2015) Fundamental Domains and Analytic Continuation of General Dirichlet Series. British Journal of Mathematics \& Computer Science, 9, 94-111.

[12] Ghisa, D. (2016) On Generalized Riemann Hypothesis II. International Journal of Scientific and Innovative Mathematical Research, 4, 46-55. https://doi.org/10.20431/2347-3142.0403006

[13] Ghisa, D. (2017) The Geometry of the Mappings by General Dirichlet Series. Advances in Pure Mathematics, 7, 1-20. https://doi.org/10.4236/apm.2017.71001

[14] Ghisa, D. and Horvat-Marc, A. (2018) Geometric Aspects of Denseness Theorems for Dirichlet Functions. British Journal of Mathematics \& Computer Science, 25, $1-11$.

[15] Ghisa, D. and Horvat-Marc, A. (2018) Geometric Aspects of Quasi-Periodic Property of Dirichlet Functions. Advances in Pure Mathematics, 8, 699-710. https://doi.org/10.4236/apm.2018.88042

[16] Speiser, A. (1934) Geometrisches zür Riemanschen Zetafunction. Mathematische 
Annalen, 110, 514-521. https://doi.org/10.1007/BF01448042

[17] Lehto, O. and Virtanen, K.I. (1973) Quasiconformal Mapping in the Plane. Springer-Verlag, Berlin. https://doi.org/10.1007/978-3-642-65513-5 